\title{
Effect of Systemic Application of Bone Marrow-Derived Mesenchymal Stem Cells on Healing of Peripheral Nerve Injury in an Experimental Sciatic Nerve Injury Model
}

\author{
Zahir KIZILAY ${ }^{1}$, Serdar AKTAS ${ }^{2}$, Nesibe KAHRAMAN CETIN ${ }^{3}$, Didem BAKAY ILHAN ${ }^{4}$, Gulfem ERSOY ${ }^{5}$, \\ Haydar Ali ERKEN ${ }^{6}$ \\ ${ }^{1}$ Adnan Menderes University, Faculty of Medicine, Department of Neurosurgery, Aydin, Turkey \\ ${ }^{2}$ Adnan Menderes University, Faculty of Medicine, Department of Pharmacology and Toxicology, Aydin, Turkey \\ ${ }^{3}$ Adnan Menderes University, Faculty of Medicine, Department of Pathology, Aydin, Turkey \\ ${ }^{4}$ Adnan Menderes University, Faculty of Medicine, Department of Biophysics, Aydin, Turkey \\ ${ }^{5}$ Adnan Menderes University, Faculty of Medicine, Department of Histology-Embryology, Aydin, Turkey \\ ${ }^{6}$ Balikesir University, Faculty of Medicine Department of Physiology, Balikesir, Turkey
}

\section{ABSTRACT}

AIM: To investigate the effects of systemic application of bone marrow-derived mesenchymal stem cells in a compression model of peripheral nerve injury.

MATERIAL and METHODS: Twenty-four male Wistar albino rats were randomly divided into 3 equal groups ( $n=8)$ : Control (C), injury (I), and stem cell and injury (SI). The sciatic nerve of the rats in the I and SI groups was subjected to clip compression for 5 minutes. Moreover, approximately $5 \times 10^{5}$ bone marrow-derived mesenchymal stem cells were given via tail vein of the rats in the SI group immediately after clip compression. The nerve conduction velocities and amplitudes of the rats were measured 30 days later. Then, the sciatic nerves were removed, and myelin damage grading and axon counting were performed. The data were analyzed by OneWay ANOVA and Tukey's post-hoc test. $P$ values less than 0.05 were considered to be statistically significant.

RESULTS: While the proximal, distal and mean latency values were higher in the I and SI groups than in the control group, the same measurements were lower in the SI group than in the I group. While the nerve conduction velocity, the amplitude of compound muscle action potential and the number of axons were lower in the I and SI groups than in the control group, the same measurements were higher in the SI group than in the I group. Moreover, myelin damage was found to be lower in the SI group than in the I group.

CONCLUSION: Systemic application of bone marrow-derived mesenchymal stem cells in a compression model of peripheral nerve injury has a positive impact on both myelin sheath and axon survival.

KEYWORDS: Bone marrow, Healing, Injury, Peripheral nerve, Mesenchymal stem cell, Nerve conductive velocity, Rats

\section{INTRODUCTION}

$\mathrm{V}$ Tarious stem cell sources have been used in many experimental studies. The stem cells used in these studies were derived from sources such as adipose tissue, umbilical cord blood, Wharton gel, bone marrow, and skin $(6,17,26,31,41)$. These stem cells were administered either locally or systemically. Thus, their effects on healing of the central and peripheral nervous system were investigated $(14,18,24)$. As a result of these studies, it has been reported that systemically injected Mesenchymal Stem Cells (MSCs) increase the synthesis and secretion of various growth factors 
by migrating to the area of injury and support both nerve cells and Schwann Cells (SCs) $(5,21,23,24,33,34)$.

Bone Marrow-Derived Mesenchymal Stem Cells (BMMSCs), which are one of the stem cell sources used in these experimental studies, can be derived from a wide range of sources such as fat tissue, peripheral blood, amniotic fluid, umbilical cord, tendons, ligaments, hair follicles, synovial membranes and fetal tissues in addition to bone marrow (13). Depending on the conditions of the experimental study, it has been shown that MSCs are converted into connective tissue series such as osteoblasts, chondrocytes, adipocytes, and myeloblasts $(25,27)$. In addition, it has also been reported that MSCs can transform into astrocytes, oligodendrocytes, microglia and neuronal cells under in vitro conditions $(1,28,32,37)$. MSCs, which are considered to be able to influence regeneration, have been used in many experimental studies in which artificial or autologous grafts were used and total nerve transections were performed $(19,31,35,36,42)$. These studies have shown that sciatic nerve regeneration and remyelination increased in the incision area $(8,22)$. It has been shown in many studies that BM-MSCs may increase peripheral nerve healing and that this effect can also be achieved by increasing the synthesis and secretion of nerve growth factor, brain-derived neurotrophic factor, ciliary neurotrophic factor, and glial cell line-derived neurotrophic factor $(5,18)$.

Although the full-thickness incisional model has been applied in most of studies investigating peripheral nerve regeneration using stem cells, the clip compression method, another model of peripheral nerve injury, has been rarely used in the models of peripheral nerve injury in which the stem cells are administered $(21,29)$. However, compression-type injury was frequently used in studies performed on the spinal cord $(4,10)$. The clip compression method was used in many experimental studies except for stem cell applications, and many substances were used to support the nerve regeneration potential $(9,16,30,39)$. The distinguishing feature of this method from the fullthickness incisional model is that the integrity of the peripheral nerve is not completely distorted. Although this provides an advantage for peripheral nerve healing, it is not a condition that prevents axon and myelin damage due to secondary damage in relation to the direct effect of compression and the duration of compression. For this reason, the clip compression method may be a more appropriate method for investigating the traps and peripheral neuropathies encountered in clinic.

In our experimental study of peripheral nerve injury, the compression was applied on the rat sciatic nerve for 5 minutes with an aneurysm clip. The efficacy of systemically injected BM-MSCs on peripheral nerve healing was histopathologically and electrophysiologically investigated. The results were discussed in the light of the literature.

\section{MATERIAL and METHODS}

\section{Animals}

All experimental protocols conducted on the animals were consistent with the National Institutes of Health Guide for the Care and Use of Laboratory Animals (National Institutes of Health Publications, No. 80-23) and were approved by the Animal Ethics committee of Adnan Menderes University (Approval Number: 64583101/2016/173). Twenty-four adult female Wistar Albino rats weighing $300 \pm 25 \mathrm{~g}$ were used in this study. All of the rats were kept in environmentally-controlled conditions at $22-25^{\circ} \mathrm{C}$ with appropriate humidity and a $12-$ hour light/dark cycle. The rats were given free access to food and water. The rats were randomly divided into 3 groups.

\section{Experimental Groups}

Group 1: Control (C), $(n=8)$; the right sciatic nerves were exposed and closed.

Group 2: Injury (I), $(n=8)$; the right sciatic nerves were exposed and nerve injury was induced by an aneurysm clip for 5 minutes.

Group 3: Stem cells and Injury (SI), $(n=8)$; the right sciatic nerves were exposed and nerve injury was induced by an aneurysm clip for five minutes. $5 \times 10^{5} \mathrm{BM}-\mathrm{MSC}$ s were injected via the tail vein just after the trauma.

\section{Generation of Right Sciatic Nerve Injury with a FE-752K Aneurysm Clip and BM-MSCs Transplantation}

Rats were anesthetized by intraperitoneal injection mix of 50 $\mathrm{mg} / \mathrm{kg}$ ketamine (Ketalar, Parke Davis, Eczacıbası Co., Istanbul, Turkey) and $10 \mathrm{mg} / \mathrm{kg}$ xylazine (Rompun, Bayer, Turkey) and allowed to breathe spontaneously. Rats were placed in the prone position and their body temperature was maintained at $37^{\circ} \mathrm{C}$. All surgical interventions were carried out on the sciatic nerve by the same surgeon by using standard microsurgery methods. The skin over the surgical site (right gluteal and femur region) was shaved and cleaned with povidone-iodine. An oblique incision was performed in the right lower extremity to remove the skin and reveal the biceps femoris in such a way to allow hip joint movement. The muscle tissue was opened and then the sciatic nerve was exposed.

In this study, an FE-752K aneurysm clip (Aesculap AG \& Co., Tutlingen, Germany) was used (29). The right sciatic nerve was compressed using a Yasargil aneurysm clip for 5 minutes, and then the clip was opened and removed. This process was applied to all the rats in group I and SI. The injured areas were also marked with 6-0 prolene suture. Then, the incision was closed in accordance with the anatomical layer. After closing the anatomical layers, BM-MSCs were applied via the tail vein for only group SI rats. The applied count of the stem cells was $5 \times 10^{5}$. The right sciatic nerves were re-exposed at the end of the $30^{\text {th }}$ day for electrophysiological measurement and histological study.

\section{Harvesting of the BM-MSCs and Culture}

Six female rats weighing 180-200 grams were used as donors to obtain BM-MSCs. Rats were sacrificed under anesthesia with ketamine (Alfamine, $10 \mathrm{mg} / \mathrm{ml}$, Netherlands) and xylazine (Alfazyne, $20 \mathrm{mg} / \mathrm{ml}$, Netherlands). The femurs of the rats were removed with sterile scissors/scalpel after sterilization of the posterior and forelegs with $70 \%$ alcohol. The removed femurs were cleared of the soft tissues and the bones were made free. Soft-tissue cleansed femurs were placed in a $50 \mathrm{ml}$ falcon tube 
(BD Falcon) containing $40 \mathrm{ml}$ of previously prepared Phosphate Buffered Saline (PBS)(Invitrogen-GIBCO, Scotland). The stem cell was transferred to the culture laboratory in a short time.

Insulin injectors drawn from a Minimum Essential Medium (MEM) (Biochrom, Berlin, Germany) containing 10\% FBS (Invitrogen-GIBCO, South America) and 1\% Penicillin-Streptomycin (Invitrogen-GIBCO) were used for this procedure. For each bone, $1 \mathrm{cc}$, and in total 5-7 cc MEM per animal was used. The femur was held in the middle and was cut from both ends of the bones with the help of scissors so that the canal was opened. Sterile falcon tubes (50 $\mathrm{ml}$ - BD Falcon) were placed in $5 \mathrm{ml}$ of medium. The curtains of the falcon tube were opened and the bones were held perpendicularly inside the falcon tube with the help of forceps. Then, the needle of the insulin injectors, which had previously been withdrawn from the medium, was injected inwardly through the channel by inserting the medium contents through the channel. The falcon tube containing the bone marrow was shaken for 5-10 seconds and then centrifuged for 5 minutes at $1250 \mathrm{rpm}$. The resulting supernatant was discarded and the medium was added to the pellet and centrifuged again at $1250 \mathrm{rpm}$ for 5 minutes. The supernatant was discarded and pipetted by adding $13 \mathrm{ml}$ of medium again onto the pellet and distributed on $25-\mathrm{cm}^{2}$ tissue culture flasks (BD-Biosciences). Thus the flask was made of cell seed. The incubation cells containing $5 \% \mathrm{CO}_{2}$ in a $37^{\circ} \mathrm{C}$ humidified medium were changed medium every three days and checked under the microscope. At the end of the third passage, the cells were counted.

\section{Flow Cytometry}

CD11b, CD29, CD45 and CD90 antibodies were used to analyze the expression of surface markers of flow cytometry MSCs. The aim here was to analyze whether suspended cells were MSCs with the help of monoclonal bodies. The MSCs' line of early passage was identified using flow cytometric analysis for surface expression markers. The MSCs attached to the surface of the culture chamber were removed from the surface with $0.25 \%$ trypsin-ethylenediamine tetraacetic acid (EDTA), transferred to tubes containing culture medium, and centrifuged. After centrifugation and washing with PBS, the pellet was resolved and the cells were suspended in PBS so as to be $1 \times 10^{6}$ after counting. Subsequently, specific Fluorescein Isothiocynate (FITC) conjugated monoclonal CD11b, CD29, CD 45 and CD90 antibodies specific to the determined cell surface markers were incubated in the dark at room temperature for 30 minutes in PBS containing 3\% BSA (as $1 \mu \mathrm{g} / \mathrm{ml}$ ). Washing solution containing $0.1 \%$ sodium azide was added after the incubation and was centrifuged at 1300 rpm for 5 minutes. The prepared cell suspension was cultured on a fluorescence-activated cell sorting (FACS) Calibur Flow Cytometry (BD Sciences) instrument and was analyzed using the BD cell QuestTM Software Program (Figure 1A-D).

\section{Cell Viability}

The cultured cells were centrifuged at $1250 \mathrm{rpm}$ for 5 minutes and separated from the petri dish after being treated with trypsin. The supernatant was discarded and the remaining pellet was pipetted with PBS. Cell counts were then performed using the developed neubauer lambda diluted $1 / 1$ with Tripan Blue.

\section{Electrophysiological Measurements}

The electrophysiological evaluation of the rat sciatic nerve was carried out under general anesthesia using a biopotential recording system (MP100, Biopac Systems Inc., Santa Barbara, CA, USA) one month after the stem cell application in order to investigate the role of stem cells in nerve regeneration. Sciatic nerve proximal \& distal latencies and base to peak amplitudes were measured. Then, conduction velocities were calculated from the measurements. For the application, the surface skin of the gastrocnemius muscle of the rats was shaved and cleaned with $70 \%$ ethanol to ensure that the data from the recording electrodes were clean. Four$\mathrm{mm}$ diameter, re-usable $\mathrm{Ag} / \mathrm{AgCl}$ surface recording electrodes (EL254S-Biopac System) were then placed on the surface of the gastrocnemius muscle with the help of electromyography (EMG) gel to obtain Compound Muscle Action Potentials (CMAP). The sciatic nerves of the rats were subjected to supramaximal electrical stimulation $(0.2 \mathrm{~ms}$ duration, $5 \mathrm{kHz}$, $50 \mathrm{~mA}, 12 \mathrm{~V} \mathrm{DC})$ three times in each measurement using a Dastre Electrode. Supramaximal stimulation was defined as a stimulus with a significantly higher value than the level needed to activate all nerve fibers. The distance between the two stimulating electrodes of the Dastre Electrode is 1.1 $\mathrm{cm}$. All stimulation and compound muscle action potential records were processed with the MP100 data acquisition system (Biopac System). For CMAP latency, the time from the stimulus artifact to the start of the CMAP was calculated. To calculate the rate of the nerve conduction, the difference of CMAP latency times recorded proximal and distal to the injured region was used. The recorded signals were analyzed with BIOPAC Acquisition Analysis Software. The conduction velocity calculations were made using the following relations:

$\square$ Latency: Proximal latency - distal latency (s)

D Distance: Proximal distance - distal distance $(\mathrm{m}): 1.1 \mathrm{~cm}$

Nerve conduction velocity: $\square$ Distance / $\square$ Latency (m / s)

\section{Sample Preparation and Histopathological Evaluation}

Nerve samples were harvested from the injured area and were placed in $10 \%$ buffered formalin solution for pathological examination. The samples were taken for routine tissue processing after the tissues were fixed in solution. Then, they were left for 14 hours in the automated tissue processing device. The tissues were fixed in paraffin and 3-4 $\mu \mathrm{m}$ sections were prepared from the paraffin blocks with a microtome. Slides prepared from the sections were stained with hematoxylin-eosin and toluidine blue for routine histopathological examination and the evaluation of axonal degeneration under $40 \times$ magnifications. The routine sections were histologically graded for axonal changes and myelin disorganization (7). Swelling (pale staining) or shrinkage (dark staining) and vacuolization were observed in the axons due to degeneration. Myelin changes including attenuation, collapse or breakdown were typically seen. Grading was performed on a scale of 0 to 3 for each section: $0=$ normal, $1=$ mild, $2=$ moderate, $3=$ severe (7). 


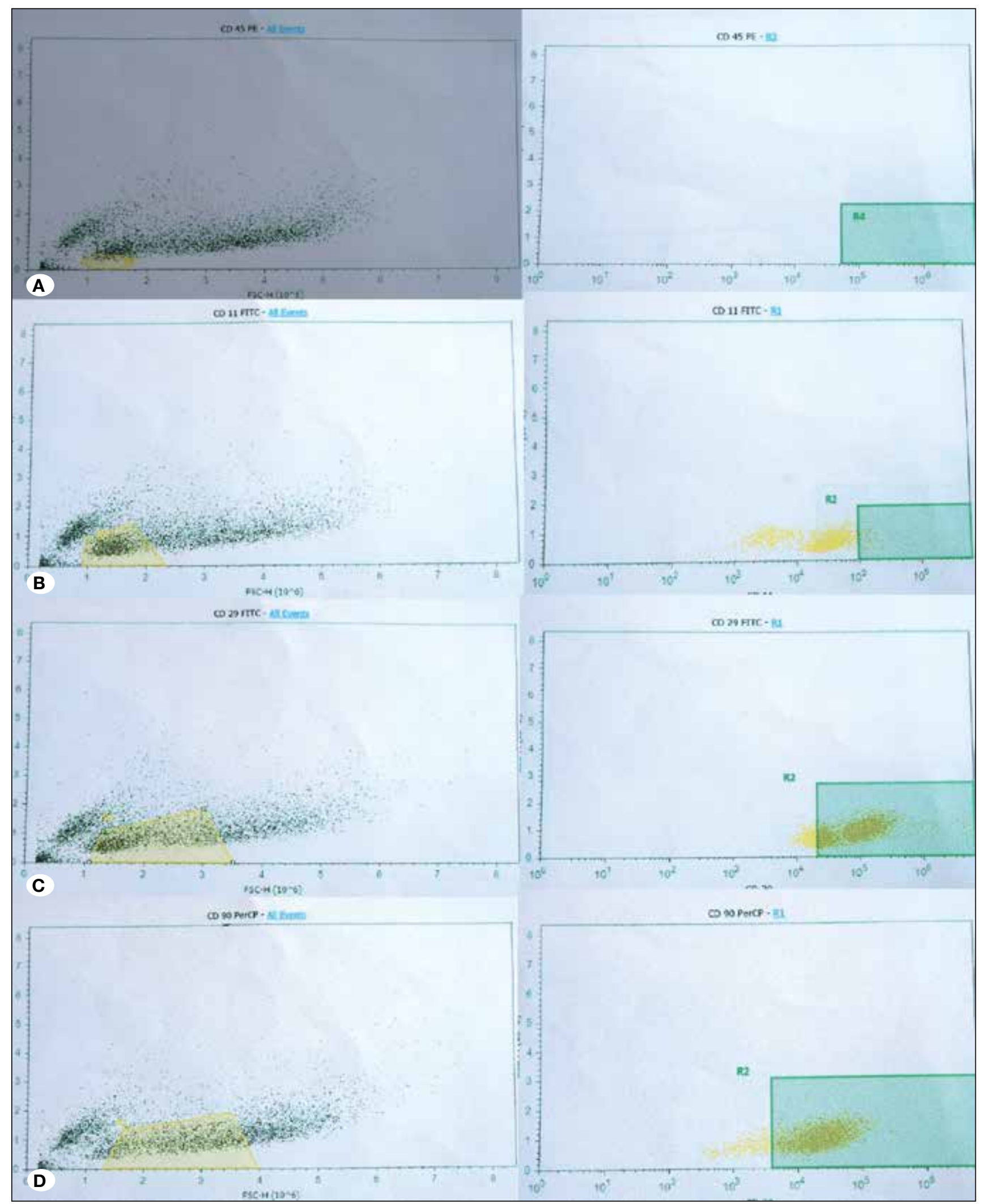

Figure 1: Flow cytometry results of immunophenotypic characteristics of MSCs isolated from rat bone marrow. The immunophenotypic characterization of the mesenchymal stem cells obtained from rat bone marrow was determined by flow cytometry technique and hematopoietic markers shown in A) CD45 PE (4.37\%), and B) CD11b FITC (2.55\%) negative stromal markers. C) CD29 FITC (82.77\%), and D) CD90 (89.31\%) positive expression. 
The numbers of the axons as well as the morphological characteristics of the axons were evaluated in cross-sections of the sciatic nerve. Five images were taken from each slide. All preparations were counted by taking samples from random areas of each sample using the DP-BSW (Microscope digital camera software) program at high magnification (x 100) under the light microscope (Olympus BX51, Olympus Co., Tokyo, Japan). This counting was made by the axon counting method that has been previously defined (38). All pathological examinations were evaluated by a pathologist blinded to the experimental group information.

\section{Statistical Analysis}

The data collected were checked for normal distribution with Shapiro-Wilk and for homogeneity of variance with Levene's Test. One-way analysis of variance was conducted to evaluate the statistical significance between the groups. Post-hoc comparisons were performed by using the Tukey test. $p$ values $<0.05$ were considered significant. The results were presented as the mean \pm SD (Standard Deviation).

\section{RESULTS}

\section{Electrophysiological Results}

The proximal (Figure 2A), distal (Figure 2B), and mean latency values (Figure $2 \mathrm{C}$ ) were found to be significantly increased in the I group compared to the $\mathrm{C}$ and SI groups. Moreover, the same measurements were found to be significantly decreased in the SI group compared to the I group $(p<0.001)$ (Table I).

Nerve conduction velocity and compound muscle action potential amplitude values (Figures 2D, E) were found to be significantly decreased in the I group compared to the $C$ and SI groups. Moreover, the same measurements were found to be significantly increased in the SI group compare to the I group $(p<0.001)$ (Table I).

\section{Histopathological Results}

While myelin degeneration was not observed in the $\mathrm{C}$ group (Figure 3A-D), myelin degeneration was markedly observed in the I (Figure 4A-D), and SI (Figure 5A-D) groups. Moreover,

Table I: Electrophysiological and Histopathological Findings of Experimental Groups (Data are Presented as mean \pm SD)

\begin{tabular}{lccc}
\hline Parameters & Control & Injury & Stem Cell and Injury \\
\hline Proximal latency (PL) (s) & $0.001304 \pm 0.00009$ & $0.002037 \pm 0.00010$ & $0.001695 \pm 0.00011$ \\
\hline Distal latency (DL) (s) & $0.001169 \pm 0.00009$ & $0.001488 \pm 0.00007$ & $0.001451 \pm 0.00011$ \\
\hline Difference between PL and DL (s) & $0.000135 \pm 0.00001$ & $0.000548 \pm 0.00008$ & $0.000243 \pm 0.00003$ \\
\hline Nerve conduction velocity (m/s) & $81.62 \pm 5.23$ & $20.50 \pm 3.20$ & $46.01 \pm 6.07$ \\
\hline Muscle compound action potential & $9.34 \pm 0.93$ & $2.17 \pm 1.10$ & $3.10 \pm 1.27$ \\
amplitude (mV) & $244.25 \pm 3.62$ & $197.83 \pm 5.74$ & $216.50 \pm 9.29$ \\
\hline Axon number & 0 & $1.83 \pm 0.41$ & $1.25 \pm 0.46$ \\
\hline
\end{tabular}

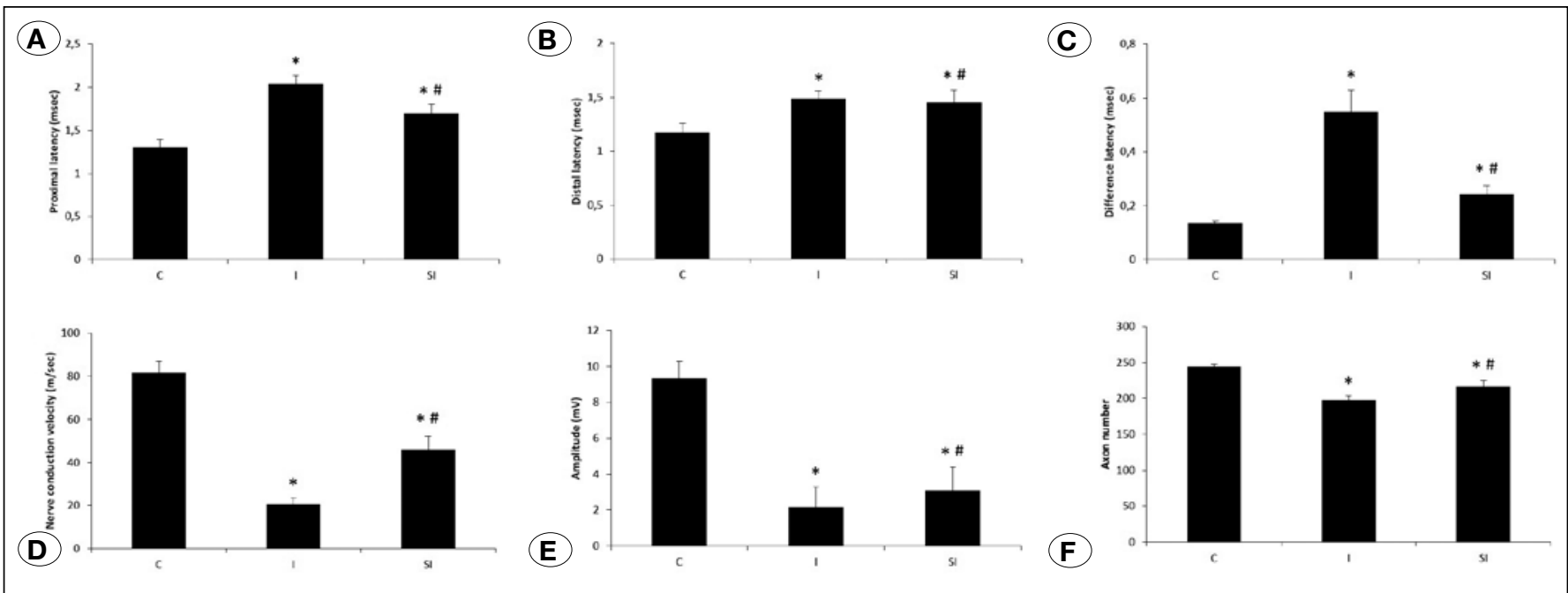

Figure 2: Electrophysiological and histological findings of experimental groups. A) Proximal latency; B) distal latency; C) difference latency; D) nerve conduction velocity; E) amplitude of compound muscle action potentials; F) axon number. C: control group; I: injury group; SI: stem cell and injury group. ${ }^{*} p<0.001$ versus $\mathrm{C}$ group. $\# \mathrm{p}<0.001$ versus I group. 
myelin degeneration was significantly lower in the SI group than the I group $(p<0.005)$ (Table I).

The number of axons was significantly lower in the SI and I groups than in the $\mathrm{C}$ group. Moreover, the number of axons was significantly higher in the SI group than in the I group $(p<0.005)$ (Figure 2F) (Table I).

\section{DISCUSSION}

When the electrophysiological results of our study were evaluated, it was seen that the application of BM-MSCs increased both CMAP and nerve conduction velocity. When we looked at the pathology results supporting these results, it was determined that the degree of axon and myelin damage was statistically significant lower in the SI group than in the I group. It is suggested that this can create the difference in nerve conduction velocity. Another histopathological finding is the fact that the number of axons was statistically significant higher in the SI group than in the I group, which may have an effect on the amplitude values.

When considered in the light of the literature, the reduced axon and myelin damage in the injured peripheral nerve with stem cell application, as seen in our study, can occur in many ways. As in the literature, the first step is that the application of stem cells increases the synthesis and secretion of various growth factors. The other step is that the applied stem cells are transformed into SCs or SCs-like cells, which is an important factor in peripheral nerve healing in the trauma area. In a study of Wang et al., it was shown that BM-MSCs increased the proliferation of SCs in the early period of trauma
( $7^{\text {th }}$ and $14^{\text {th }}$ days). In addition, it was demonstrated that they increased axon growth, expression of neural skeletal molecules, and neurotrophic factors and their receptors on the regenerating nerves of rats in the trauma area in the early period (34). Another study conducted by Han et al. reported that peripheral nerve healing in rats with diabetic neuropathy might be due to the fact that BM-MSC application directly increases angiogenesis, has neurotrophic effects and restores myelinization in the nerve. Moreover, in the same study, BMMSCs were almost never converted into SCs (11). A study carried out by Zarbakhsh et al. and examined the effects of Bone Marrow-Derived Stromal Stem Cells (BM-SCs) on peripheral nerve healing when used alone or with SCs (42). The conclusion of this study was that the use of BM-SCs and SCs separately or in combination had a positive effect on peripheral nerve healing. A similar study was performed by Matthes et al. and compression-type injury was created, and BM-SCs were injected into the systemic circulation via the femoral vein. As a result of this study, it was shown that systemically injected stem cells were settled in the proximal and distal area of the injured sciatic nerve of rats in the BM-SCs group and increased nerve regeneration. However, in the same study, it could not be disclosed how they could provide this effect and what effect they might have on the number of axons (21). When our study and other studies in the literature are considered together, it is possible to conclude that BM-MSCs migrate to the injury area in the early recovery period of the injured peripheral nerve, and enhance the synthesis of various neurotrophic or growth factors, rather than transforming into SCs or SCs-like cells. Thus, it is possible that they promote remyelination in partially damaged axons by supporting nerve

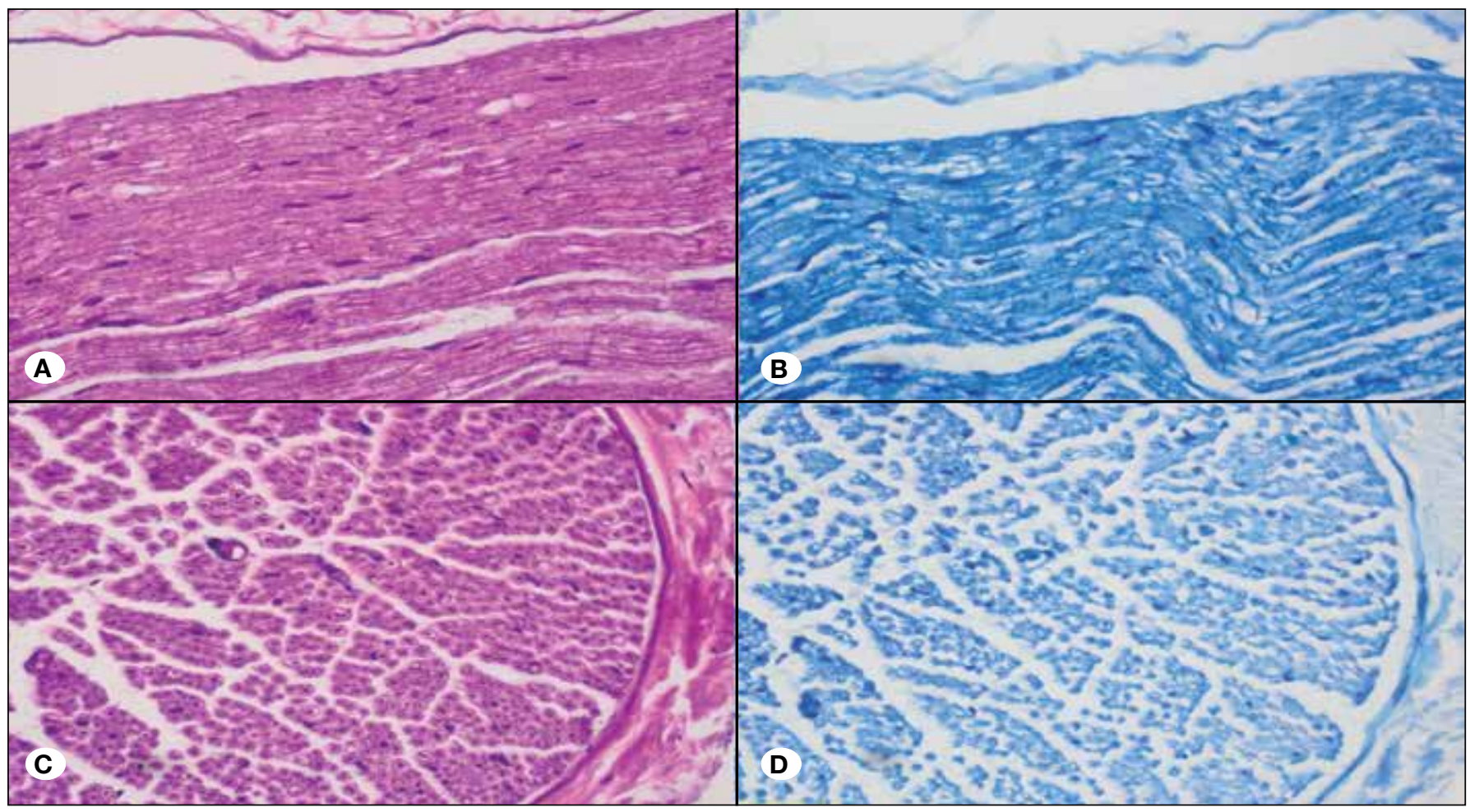

Figure 3: Normal architecture of sciatic nerve of rat is seen at longitudinal (A,B), and axial (C,D) sections of control group hematoxylineosin (A,C), and toluidine blue (B,D) staining. (x40 magnification). 


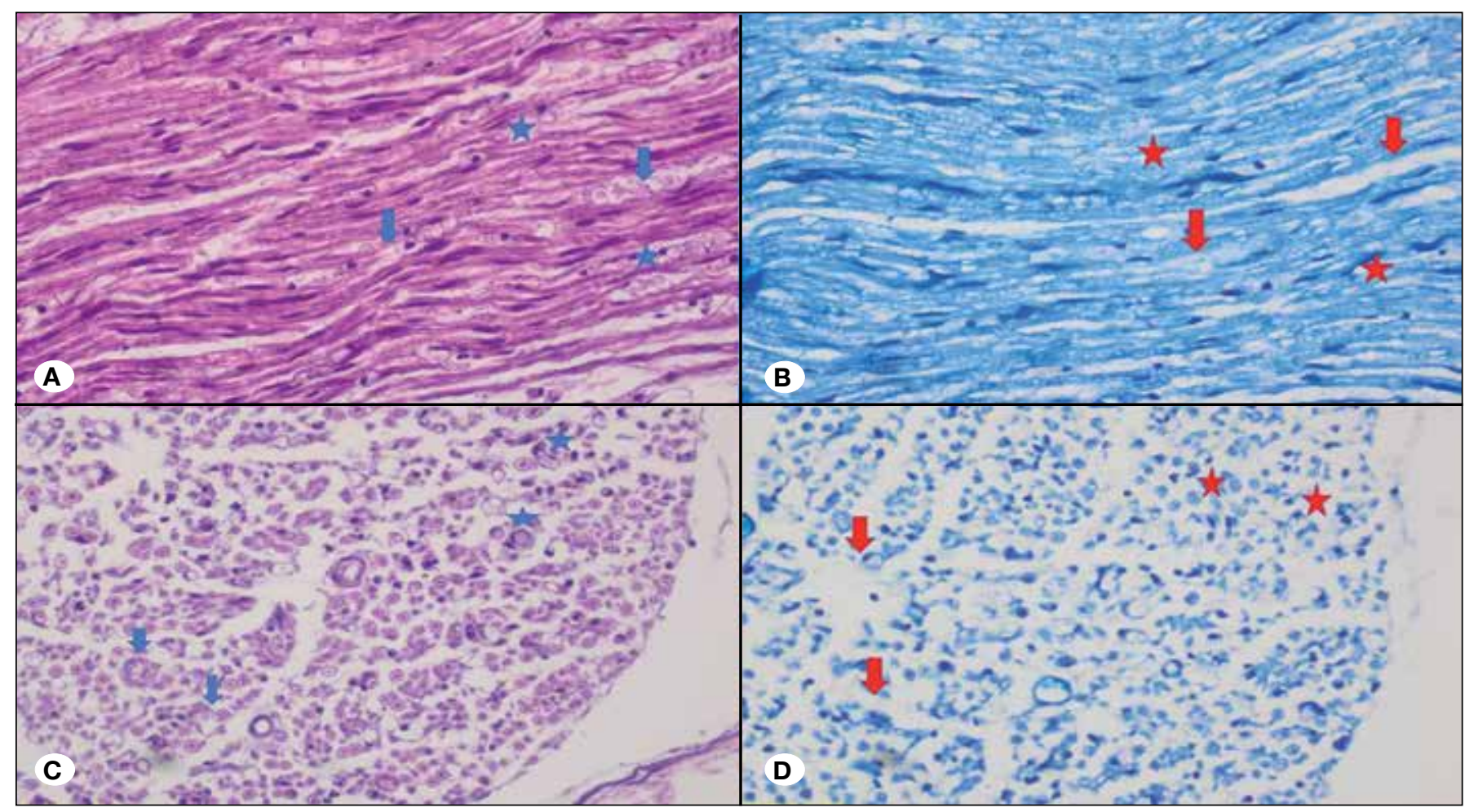

Figure 4: Mild axonal vacuolization (asterisks) and myelin destruction (arrows) of sciatic nerve of rat is seen at longitudinal (A,B), and axial $(\mathbf{C}, \mathbf{D})$ sections of stem cell and injury group hematoxylin-eosin (A,C), and toluidine blue (B,D) staining. (x40 magnification).

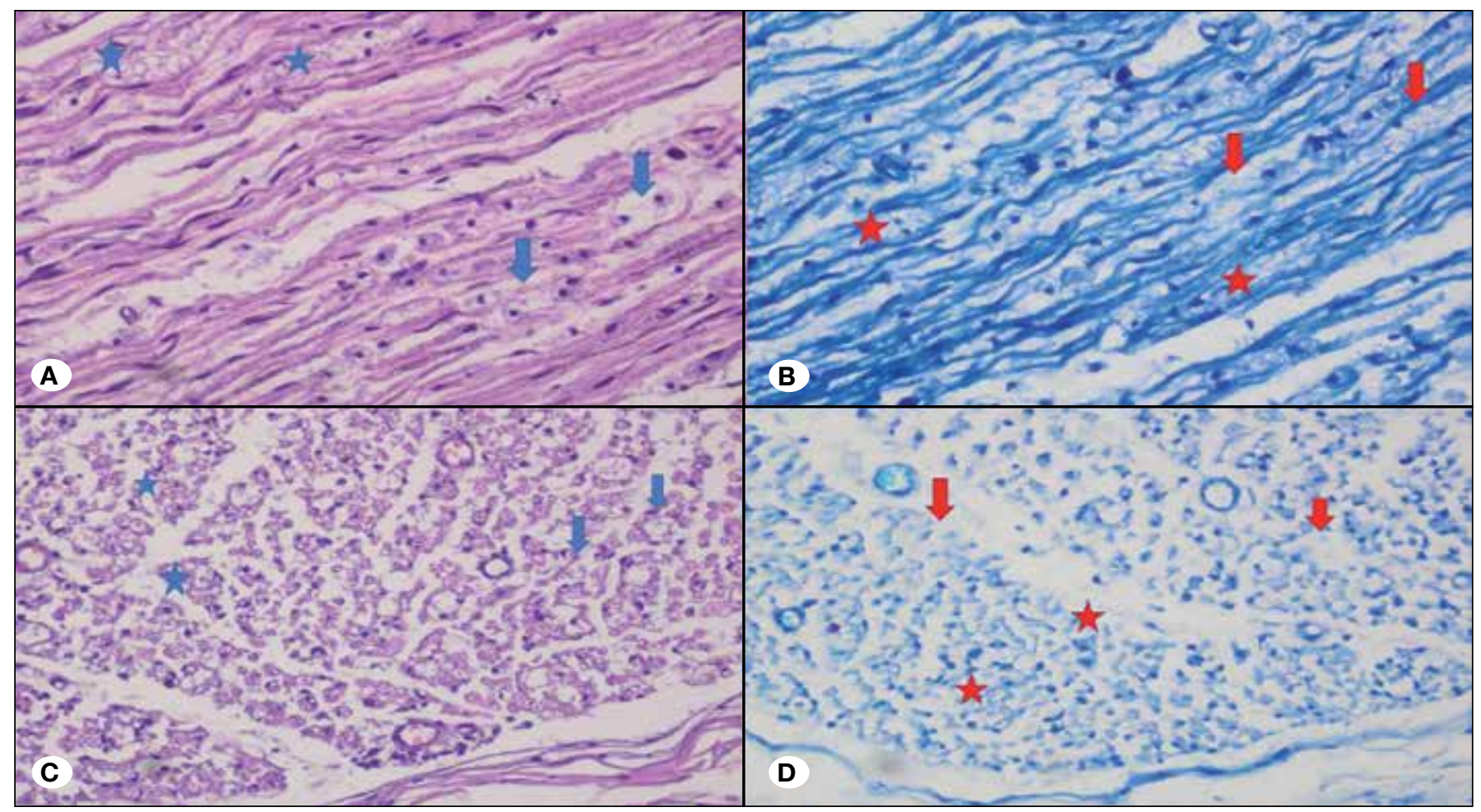

Figure 5: Increased axonal vacuolization (asterisks), and myelin destruction (arrows) of sciatic nerve of rat is seen at longitudinal (A,B), and axial (C,D) sections of injury group hematoxylin-eosin (A,C), and toluidine blue (B,D) staining. (x40 magnification). 
cells and SCs. Our study also revealed that the application of BM-MSCs after injury reduced myelin damage in the axons and this information supports the literature.

Another result of our study is that the application of BM-MSCs after injury in compression-type peripheral nerve trauma had a positive effect on the number of surviving axons. When the mechanism of compression-type trauma is examined, the primary cause of trauma is damage due to the direct effect of the compression. The secondary cause of trauma is reperfusion injury resulting from the removal of the compression after mechanical compression on blood vessels feeding the nerves during compression. The development of secondary damage following primary damage can increase the damage in injured axons, which can lead to loss of axons. When the literature is examined, it is observed that researchers have shown that nerve growth factors can provide retrograde stimulation in the peripheral nerve, which can protect central neurons (3). In addition, other researchers have shown that the application of exogenous brain-derived neurotrophic factor in the model of amyotrophic lateral sclerosis increased the expression in the anterior horn of the spinal cord and that this increased the survival rate of the cells at a statistically significant level $(2,15,20)$. When the efficacy of the stem cell applications in ischemic reperfusion injury is examined, it is observed that Inan et al. have shown that the levels of interleukin $1 \beta$ (IL-1 $\beta$ ), transforming growth factor- $1 \beta$, tumor necrosis factor- $\alpha$ and IL-6, which are pro-inflammatory cytokines, decreased in the stem cell treatment groups after the application of MSCs in ischemic reperfusion injury. In the same study, it was shown that MSCs could promote antioxidant enzymes, which can decrease oxidative stress by reducing MDA levels (12). Yin et al. reported that administration of MSCs in a spinal cord ischemia-reperfusion injury showed an anti-apoptotic activity on the medulla spinalis and that it might protect the spinal cord against ischemic-reperfusion injury (40). When our study and these studies are considered together, it is possible to conclude that the application of BM-MSCs after injury in a compression-type trauma model promotes SCs and axons via growth factors and thereby may reduce directly or indirectly the effect of primary damage responsible for apoptosis and secondary damage due to oxidative stress. This may limit the mechanism of apoptosis responsible for the loss of axons.

In this study, the application of BM-MSCs was shown for the first time to have positive effects on the healing of the peripheral nerve after a compression-type peripheral nerve injury model. However, there are a few limitations in the study. Firstly, the study had a limited number of rats. Secondly, different number of stem cells and durations should have been investigated. Thirdly, ultrastructural differences and functional evaluations should have been investigated as well.

\section{- CONCLUSION}

Systemic application of BM-MSCs after compression-type peripheral nerve injury might have statistically significant electrophysiological and histopathological effects on peripheral nerve healing. Further studies are required in order to reveal the mechanisms of the results found in this study.

\section{REFERENCES}

1. Azizi SA, Stokes D, Augelli BJ, DiGirolamo C, Prockop DJ: Engraftment and migration of human bone marrow stromal cells implanted in the brains of albino rats-similarities to astrocyte grafts. Proc Natl Acad Sci U S A 95(7):3908-3913, 1998

2. Azzouz M, Ralph GS, Storkebaum E, Walmsley LE, Mitrophanous KA, Kingsman SM, Carmeliet $P$, Mazarakis ND: VEGF delivery with retrogradely transported lentivector prolongs survival in a mouse ALS model. Nature 429(6990): 413-417, 2004

3. Baumgartner BJ, Shine HD: Permenant rescue of lesioned neonatal motoneurons and enhanced axonal regeneration by adenovirus-mediated expression of glial cell-line-derived neurotrophic factor. J Neurosci Res 54(6):766-777, 1998

4. Boido M, Garbossa D, Fontanella M, Ducati A, Vercelli A: Mesenchymal stem cells transplantation reduces glial cyst and improves functional outcome after spinal cord injury. World Neurosurg 81(1):183-190, 2014

5. Chen CJ, Ou YC, Liao SL, Chen YW, Chen SY, Wu CW, Wang CC, Wang WY, Huang YS, Hsu SH: Transplantation of bone marrow stromal cells for peripheral nerve repair. Exp Neurol 204(1):443-453, 2007

6. Chen Z, Pradhan S, Liu C, Le LQ: Skin derived precursors as a source of progenitors for cutaneous nerve regeneration. Stem Cells 30(10):2261-2270, 2012

7. Coban YK, Cirakli H, Kurulas EB: Ischemic preconditioning reduces the severity of ischemia-reperfusion injury of peripheral nerve in rats. J Brachial Plex Peripher Nerve Inj 1:2, 2006

8. Dezawa M, Takahashi I, Esaki M, Takano M, Sawada H: Sciatic nerve regeneration in rats induced by transplantation of in vitro differentiated bone marrow stromal cells. Eur $\mathrm{J}$ Neurosci 14(11):1771-1776, 2001

9. Emel E, Ergün SS, Kotan D, Gürsoy EB, Parman Y, Zengin A, Nurten A: Effects of insulin like growth factor-I and plateletrich plasma on sciatic nerve crush injury in a rat model. $J$ Neurosurg 114(2):522-528, 2011

10. Gao S, Ding J, Xiao HJ, Li ZQ, Chen Y, Zhou XS, Wang JE, Wu J, Shi WZ: Anti-inflammatory and anti-apoptotic effect of combined treatment with methylprednisolone and amniotic membrane mesenchymal stem cells after spinal cord injury. Neurochem Res 39(8):1544-1552, 2014

11. Han JW, Choi D, Lee MY, Huh YH, Yoon YS: Bone marrowderived stem cells improve diabetic neuropathy by both angiogenesis and myelination in peripheral nerves. Cell Transplant 25:313-326, 2016

12. Inan M, Bakar E, Cerkezkayabekir A, Sanal F, Ulucam E, Subasi C, Karaoz E: Mesenchymal stem cells increase antioxidant capacity in intestinal ischemia/reperfusion damage. J Pediatr Surg 52(7):1196-1206, 2017

13. Jiang L, Jones $S$, Jia X: Stem cell transplantation for peripheral nerve regeneration: Current options and opportunities. Int $J$ Mol Sci 18(1): E94, 2017

14. Kao $\mathrm{CH}$, Chen $\mathrm{SH}$, Chio CC, Lin MT: Human umbilical cord blood-derived CD34+ cells may attenuate spinal cord injury by stimulating vascular endothelial and neurotrophic factors. Shock 29(1):49-55, 2008 
15. Kaspar BK, Llado J, Sherkat N, Rothstein JD, Gage FH: Retrograde viral delivery of IGF-1 prolongs survival in a mouse ALS model. Science 301(5634):839-842, 2003

16. Kato K, Liu H, Kikuchi S, Myers RR, Shubayev VI: Immediate anti-tumor necrosis factor alpha (etanercept) therapy enhances axonal regeneration after sciatic nerve crush. J Neurosci Res 88(2):360-368, 2010

17. Kingham PJ, Kalbermatten DF, Mahay D, Armstrong SJ, Wiberg M, Terenghi G: Adipose-derived stem cells differentiate into schwann cell phenotype and promote neurite outgrowth in vitro. Exp Neurol 207(2):267-274, 2007

18. Liu G, Cheng Y, Guo S, Feng Y, Li Q, Jia H, Wang Y, Tong $\mathrm{L}$, Tong $\mathrm{X}$ : Tranplantation of adipose-derived stem cells for peripheral nerve repair. Int J Mol Med 28(4):565-572, 2011

19. Liu GB, Cheng YX, Feng YK, Pang CJ, Li Q, Wang Y, Jia H, Tong XJ: Adipose-derived stem cells promote peripheral nerve repair. Arch Med Sci 7(4):592-596, 2011

20. Lu YY, Wang LJ, Muramatsu S, Ikeguchi K, Fujimoto K, Okada T, Mizukami H, Hanazono Y, Kume A, Nagatsu T, Ozawa K, Nakano I: Intramuscular injection of AAV-GDNF results in sustained expression of transgenic GDNF, and its delivery to spinal motoneurons by retrograde transport. Neurosci Res 45 (1):33-40, 2003

21. Matthes SM, Reimers K, Janssen I, Liebsch C, Kocsis JD, Vogt PM, Radtke C: Intravenous transplantation of mesenchymal stromal cells to enhance peripheral nerve regeneration. Biomed Res Int 2013: 573169, 2013

22. Mimura T, Dezawa M, Kanno $H$, Sawada $H$, Yamamoto I: Peripheral nerve regeneration by transplantation of bone marrow stromal cell-derived schwann cells in adult rats. J Neurosurg 101(5):806-812, 2004

23. Nijhuis TH, Bodar CW, van Neck JW, Walbeehm ET, Siemionow M, Madajka M, Cwykiel J, Blook JH, Hovius SE: Natural conduits for bridging a $15 \mathrm{~mm}$ nerve defect: Comparison of the vein supported by muscle and bone marrow stromal cells with nerve autograft. J Plast Reconstr Aesthet Surg 66(2): 251-259, 2013

24. Osaka M, Honmou Q, Murakami T, Nonaka T, Houkin K, Hamada H, Kocsis JD: Intraveneous administration of mesenchymal stem cells derived from bone marrow after contusive spinal cord injury improves functional outcome. Brain Res1343:226-235, 2010

25. Paniushin OV, Domaratskaia EL, Starostin VI: Mesenchymal stem cells: Sources, phenotype, and differentiation potential. Iz Akad Nauk Ser Biol 1:6-25, 2006 (In Russian)

26. Peng J, Wang Y, Zhang L, Zhao B, Zhao Z, Chen J, Guo Q, Liu S, Sui X, Xu W, Lu S: Human umbilical cord wharton's jellyderived mesenchymal stem cells differentiate into schwann cell phenotype and promote neurite outgrowth in vitro. Brain Res Bull 84(3):235-243, 2011

27. Pittenger MF, Mackay AM, Beck SC, Jaiswal RK, Douglas R, Mosca JD, Moorman MA, Simonetti DW, Craig S, Marshak DR: Multilineage potential of adult human mesenchymal stem cells. Science 284(5411):143-147, 1999

28. Sanchez-Ramos J, Song S, Cardozo-Pelaez F, Hazzi C, Stedeford T, Willing A, Freeman TB, Saporta S, Janssen W, Patel N, Cooper DR, Sanberg PR: Adult bone marrow stromal cells differentiate into neural cells in vitro. Exp Neurol 164(2): 247-256, 2000
29. Sarikcioglu L, Ozkan O: Yasargil-phynox aneurysm clip: A simple and reliable device for making a peripheral nerve injury. Int J Neurosci 113(4):455-464, 2013

30. Serarslan Y, Bal R, Altug ME, Kontas T, Keles ON, Unal D, Unal $B$ : Effects of trimetazidine on crush injury of the sciatic nerve in rats: Biochemical and stereological study. Brain Res 1247: 11-20, 2009

31. Sung MA, Jung HJ, Lee JW, Lee JY, Pang KM, Yoo SB, Alrashdan MS, Kim SM, Jahng JW, Lee JH: Human umbilical cord blood-derived mesenchymal stem cells promote regeneration of crush-injured rat sciatic nerves. Neural Regen Res 7(26):2018-2027, 2012

32. Tohill M, Mantovani C, Wiberg M, Terenghi G: Rat bone marrow mesenchymal stem cells express glial markers and stimulate nerve regeneration. Neurosci Lett 362(3):200-203, 2004

33. Vaquero J, Zurita M, Oya S, Santos M: Cell therapy using bone marrow stromal cells in chronic paraplegic rats: Systemic or local administration? Neurosci Lett 398(1-2):129-134, 2006

34. Wang J, Ding F, Gu Y, Liu J, Gu X: Bone marrow mesenchymal stem cells promote cell proliferation and neurothropic function of schwann cells in vitro and in vivo. Brain Res 1262:7-15, 2009

35. Wang Y, Jia H, Li WY, Tong XJ, Liu GB, Kang SW: Synergistic effects of bone mesenchymal stem cells and chondroitinase $\mathrm{ABC}$ on nerve regeneration after acellular nerve allograft in rats. Cell Mol Neurobiol 32(3):361-371, 2012

36. Wang Y, Li ZW, Luo M, Li YJ, Zhang KQ: Biological conduits combining bone marrow mesenchymal stem cells and extracellular matrix to treat long-segment sciatic nerve defects. Neural Regen Res 10(6):965-971, 2015

37. Woodbury D, Schwarz EJ, Prockop DJ, Black IB: Adult rat and human bone marrow stromal cells differentiate into neurons. J Neurosci Res 61(4):364-370, 2006

38. Yang I, Bashaw GJ: Son of the sevenless directly links the Robo receptor to rac activation to control axon repulsion at the midline. Neuron 52:595-607, 2006

39. Yildirim AE, Dalgic A, Divanlioglu D, Akdag R, Cetinalp NE, Alagoz F, Helvacioglu F, Take G, Guvenc Y, Koksal I, Belen $A D$ : Biochemical and histopathological effects of catechin on experimental peripheral nerve injuries. Turk Neurosurg 25(3): 453-460, 2015

40. Yin F, Guo L, Meng CY, Liu YJ, Lu RF, Li P, Zhou YB: Transplantation of mesenchymal stem cells exerts antiapoptotic effects in adult rats after spinal cord ischemiareperfusion injury. Brain Res 1561:1-10, 2014

41. Zarbakhsh S, Goudarzi N, Shirmohammadi M, Safari M: Histological study of bone marrow and umbilical cord stromal cell transplantation in regenerating rat peripheral nerve. Cell $\mathrm{J}$ 17(4):668-677, 2016

42. Zarbakhsh S, Moradi F, Joghataei MT, Bahktiari M, Monsouri $\mathrm{K}$, Abedinzadeh $\mathrm{M}$ : Evaluation of the functional recovery in sciatic nerve injury following the co-transplantation of schwann and bone marrow stromal cells in rats. Bas Clin Sci $4(4): 291-298,2013$ 